\title{
Análisis de eficiencia hospitalaria en Chile
}

Analysis of hospital efficiency in Chile

\author{
Planck Barahona-Urbina \\ Doctor en Economía. \\ Profesor, Facultad de Ingeniería, Departamento de Industria y Negocios, Universidad de Atacama, Chile.
}

\begin{abstract}
Resumen
Introducción: La importancia del análisis envolvente de datos (AED) radica en poder determinar cuáles de los hospitales regionales presentan un mejor desempeño relativo y cuáles podrían mejorar el uso de los recursos. Objetivos: Analizar, haciendo uso de la técnica no paramétrica de análisis envolvente de datos, la eficiencia técnica hospitalaria en Chile. Diseño: Análisis de tipo no paramétrico. Lugar: Hospitales públicos regionales de Chile. Intervenciones: Aplicación del AED para la evaluación de la eficiencia de los establecimientos de la salud. Materiales: Se utilizó datos para el período comprendido entre 1997 y 2006 de las doce regiones del país más la región Metropolitana (RM), de 190 hospitales públicos de distintos grados de especialización. Se identificó datos de actividad hospitalaria y personal facultativo, esto es, como inputs a médicos, enfermeras, matronas, camas disponibles, y como variable output el egreso hospitalario. Principales medidas de resultados: Eficiencia hospitalaria. Resultados: En el año 1997, las regiones IV, VII, VIII, y IX fueron calificadas como técnicamente eficientes, Io que ha significado que han hecho un adecuado uso de sus recursos. Conclusiones: En diez años de estudio, 1997 a 2006, únicamente en el año 1997 cuatro regiones de Chile fueron eficientes. Los hospitales regionales deberían reducir sus inputs en promedio un $10 \%$ para situarse sobre la frontera eficiente. El análisis DEA es una buena herramienta para llevar a cabo mediciones de eficiencia y evaluar resultados de gestión, pues permite conocer aquellas unidades que realizan la mejor asignación de sus recursos en comparación al resto de la muestra.
\end{abstract}

Palabras clave: Hospital, eficiencia técnica global, análisis envolvente de datos (AED).

Abstract

Introduction: Data envelopment analysis (DEA) helps determining which of the regional hospitals have better relative performance and which could improve the use of resources. Objectives: To analyze, using data envelopment analysis (DEA), hospitals technical efficiency in Chile. Design: Non parametric analysis. Setting: Chile's Regional Public Hospitals. Interventions: Application of DEA to determine the efficiency of health establishments. Materials: Data for the period 1997-2006 of 190 public hospitals of different levels of expertise of the twelve regions of the country and the Metropolitan Region. Hospital and medical staff activity data have been identified -doctors, nurses, midwives, beds available as inputs and hospital discharge as output. Main outcome measures: Hospitals efficiency. Results: In 1997 regions IV, VII, VIII and IX hospitals were classified as technically efficient, that is, have made adequate use of their resources. Conclusions: In the ten-year study, 1997-2006, only in 1997 four Chile regions were efficient. Regional hospitals should reduce their inputs an average of $10 \%$ in order to stand on the efficient frontier. DEA is a good tool to measure efficiency and evaluate results of management.

Keywords: Hospital, global technical efficiency, data envelopment analysis (DEA).

An Fac med. 2011;72(1):33-8

\section{INTRODUCCIÓN}

La evaluación de la eficiencia a nivel hospitalario tiene relación con el uso eficiente de los recursos. En un mundo donde los recursos son escasos, la eficiencia implica o es el equivalente al empleo de los recursos de manera adecuada, con el objetivo de obtener el máximo beneficio. Se dice que una actividad productiva es eficiente si se reduce la cantidad utilizada de algún input sin perjuicio de la cantidad obtenida o si se puede aumentar la producción sin utilizar mayores cantidades de input ${ }^{(1)}$. El uso de técnicas para el análisis de eficiencia hospitalaria ha permitido que los sectores de salud público y privado permitan reevaluar la gestión de su organización para un mejor uso de sus recursos. Este tipo de información permitiría la intervención a priori en los centros con un comportamiento anómalo respecto del resto de establecimientos; a posteriori, además, se podría intervenir estableciendo premios o penalizaciones asociadas a mejoras o retrocesos en el nivel de productividad (2). El mal uso de recursos es el común denominador en los hospitales públicos de Chile y ello hace necesaria relacionar los costos de los inputs (insumos) empleados con los outputs (productos) obtenidos.

La evaluación de la eficiencia de los servicios hospitalarios es una actividad prioritaria para los responsables de los servicios sanitarios. Una de las técnicas empleadas para dicho análisis ha sido el empleo del modelo no paramétrico análisis envolvente de datos (AED), una aplicación de programación lineal muy valiosa en la realización de análisis 
comparativos de eficiencia, particularmente en el sector público ${ }^{(3)}$. La característica fundamental de esta técnica es que no postula una forma funcional para los datos, es decir, no impone una forma paramétrica para la función de producción. Por lo tanto, no existe error de especificación, lo que hace de este método muy atractivo para los investigadores. Las investigaciones que utilizan modelos de frontera se han hecho muy comunes en el análisis de eficiencia hospitalaria.

Un estudio realizado en Andalucía, España, llevó a cabo un análisis de eficiencia en los servicios de medicina preventiva de dichos hospitales. Para el estudio seleccionaron siete hospitales andaluces, calculándose la eficiencia relativa de las siete unidades. Para ello, se escogió dos inputs (salario mensual del personal médico, salario de personal no médico) y tres outputs (formación médico interno residente -MIR- de la especialidad, inmunoprofilaxis, exámenes de salud realizados). De los siete servicios estudiados, tres mostraron algún tipo de ineficiencia relativa (eficiencia $<1$ ). En este sentido, un servicio es identificado como ineficiente cuando fracasa en alcanzar los mejores niveles posibles de eficiencia de outputs y/o cuando emplea excesiva cantidad de inputs ${ }^{(4)}$. En la misma línea de investigación, se llevó a cabo análisis de eficiencia técnica de los establecimientos de salud en Lima, Perú, agrupados en microrredes, bajo la estimación de una frontera eficiente. Para el estudio se consideró tres inputs (personal médico, otro personal y consumo de medicamentos) y dos outputs (atenciones y actividades preventivas), midiéndose la eficiencia técnica global, para posteriormente medir la eficiencia técnica pura. Los resultados identificaron que nueve microrredes tuvieron nivel de eficiencia técnica global de $100 \%$, mientras que las restantes se mantuvieron bajo el nivel de eficiencia. La eficiencia media de las microrredes estudiadas fue $97,9 \%$, lo que mostró que para alcanzar el nivel de eficiencia tendrían que reducir sus inputs en un $3 \%{ }^{(5)}$.
En un estudio similar realizado en el estado de Virginia, en los Estados Unidos, se analizó en una muestra de 53 hospitales la eficiencia en el cuidado de enfermedades agudas, utilizando la técnica análisis envolvente de datos (AED). Como medida de output se incluyó el total de pacientes dados de alta, total de visitas de pacientes externos provenientes de emergencias durante el año 2003 y formación médica a tiempo completo, incluyendo prácticas médicas y odontológicas. Como inputs se utilizó el tamaño del hospital, la demanda (cantidad de gastos operacionales, capital o gastos de depreciación), el personal a tiempo completo o medio tiempo y el total de activos. El estudio determinó que 32 hospitales tuvieron mal desempeño en lo que respecta a la eficiencia técnica y calidad ${ }^{(6)}$. Otros estudios han hecho uso de la técnica AED para determinar la eficiencia de las prácticas médicas (7-9), en el sentido de más o menos días de hospitalización y la eficiencia de los servicios en el contexto de nuevas formas organizativas ${ }^{(10)}$. Como vemos, las aportaciones empíricas en el ámbito del estudio de la eficiencia hospitalaria son abundantes y heterogéneas en su forma de aplicación, debido al interés por conocer la mejor combinación de recursos y mejorar la gestión de los centros hospitalarios. Con este tipo de análisis se puede identificar las mejores y peores prácticas asociadas con una eficiencia y productividad elevada o reducida, respectivamente.

En este contexto, Chile ha realizado una serie de reformas, como la implementación de nuevos mecanismos de pago a proveedores (en el ámbito que compete a médicos u hospitalarios) -con el objetivo de contener costes-y la referida al uso eficiente de los recursos mejorando la gestión de las organizaciones hospitalarias. Este tipo de reformas tiene que ver con el creciente incremento de los gastos en salud (pasando de un 5,3\% del PIB en el año 1995 a 6,9\% en el año 2008). Las causas del crecimiento del gasto sanitario son muy diversas, y su origen podría estar en el progreso científico y técnico de la medicina y en el aumento de la demanda de asistencia médica, estimulada por factores tan variados como la mayor cultura sanitaria de la población, mayor confianza en la medicina, niveles de vida más altos, el deseo de la sociedad actual de disfrutar de la vida de la mejor forma y más ampliamente posible y, sobre todo, por el envejecimiento progresivo de la población, que por sí mismo aumenta el consumo de medicamentos y de asistencia médica.

El objetivo de este trabajo es evaluar la eficiencia técnica y de escala de los hospitales públicos en Chile, haciendo uso de la técnica análisis envolvente de datos (AED).

\section{MÉTODOS}

La fuente de datos fue proporcionada por el Departamento de Estadística e Información del Ministerio de Salud de Chile (MINSAL), así como de la División de Gestión de las Personas (Subsecretaria de redes asistenciales). Para nuestra investigación hemos utilizado datos de actividad hospitalaria y personal facultativo de los años 1997 hasta 2006, de las 11 regiones del país más la región metropolitana (Santiago), para un total de 190 hospitales públicos con distintos grados de especialización. Determinamos como variables inputs a los médicos, enfermeras, matronas, camas disponibles y, como variable output, el egreso hospitalario. Respecto de esta última variable, se entiende que el objetivo de una institución hospitalaria prestadora de servicios de salud es producir egresos como resultado final. El egreso se entiende como el tratamiento brindado al paciente que ha ingresado al hospital con alguna patología, en aras de mejorar su estado de salud, utilizando para ello una mezcla de recursos de orden diagnóstico, terapéutico, médico o quirúrgico y recursos tecnológicos, dependiendo del grado de complejidad de la institución. Esta variable ha sido utilizada como proxy del volumen de pacientes que la institución atiende en servicios no ambulatorios, exceptuando 


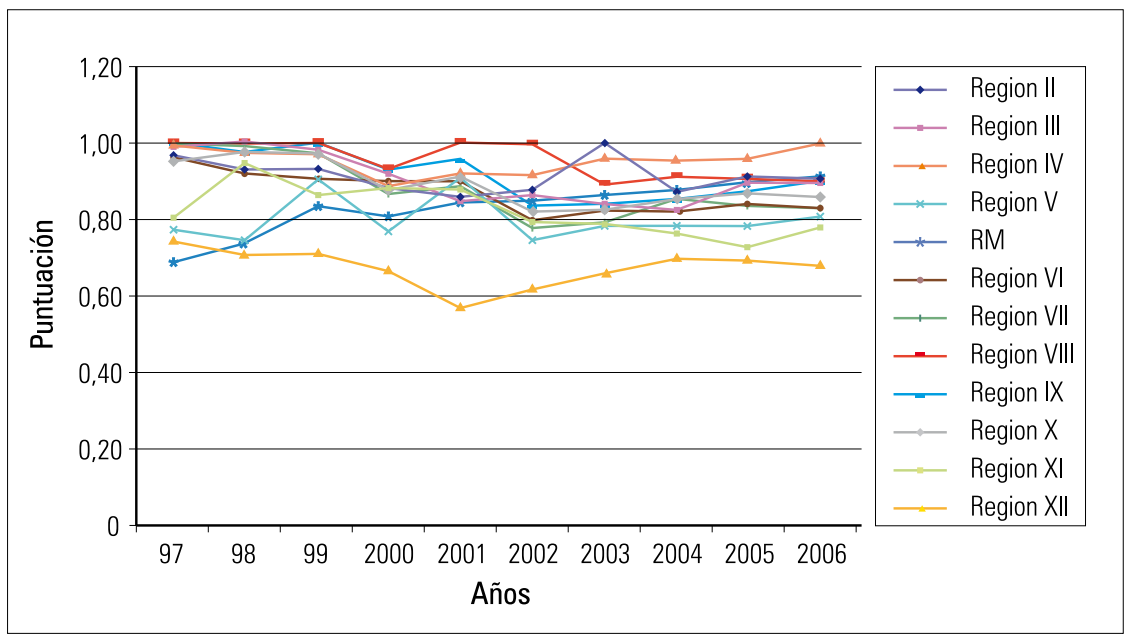

Figura 1. Eficiencia técnica global (modelo AED-CCR). Fuente: Elaboración propia.

partos. Se asume que una institución tratará de maximizar sus egresos con una utilización eficiente de sus recursos, es decir, hacer un mejor uso posible de los recursos limitados a los agentes económicos. En síntesis, hemos utilizado cuatro inputs y un output, evaluadas en hospitales de 12 regiones de Chile, lo que nos da una muestra de $60 \mathrm{ob}$ servaciones a lo largo de un período de 10 años.

Para evaluar la diferencia de eficiencia productiva entre unidades hemos usado el método análisis envolvente de datos (AED), un método de programación matemática que convierte múltiples inputs y outputs medidos en una sola suma de productividad eficiente. El AED está basado en la eficiencia relativa ${ }^{(11,12)}$. Es decir, los autores de esta herramienta flexibilizan el supuesto de AED en la cual se asumía rendimientos constantes de escala (DEA-CCR), permitiendo que la topología de rendimiento a escala se caracterice por una tecnología variable -constante, creciente o decreciente-. Por consiguiente, para la medición de la eficiencia técnica seguiremos el método técnica (AED) orientación inputs ${ }^{(1)}$, asumiendo rendimientos constantes de escala ${ }^{(2)}$, que nos ayudarán a construir lo que se denomina superficie envolvente, frontera eficiente o función de producción del conjunto de entidades que están siendo objeto de análisis. Aquellas entidades que determinan la envolvente la denominaremos entidades eficientes y permitirán la evaluación de la eficiencia relativa de cada una de las entidades. Una de las ventajas del $A E D$ es que, por ser una técnica no-paramétrica ${ }^{(3)}$, no supone alguna forma funcional de la relación entre los inputs y los outputs ni da por existente una distribución de la ineficiencia $^{(13)}$, requiriendo tan solo algunas propiedades formales como en el conjunto de producción, tales como convexidad y libre disposición de los inputs y outputs, que permitan la aplicación de las técnicas de programación lineal en que se basa. Formalmente, el cálculo del puntaje de productividad eficiente, según el modelo DEA-CCR (cálculo de la eficiencia técnica global), se efectúa solucionando el siguiente problema de programación lineal. Sea $\mathrm{N}$ el número de regiones a analizar, las cuales utilizan una cantidad determinada de $\mathrm{M}$ inputs para producir S outputs. Para la i-ésima región, se obtiene una matriz $x$ de tamaño $(M x 1)$ inputs y una matriz $y$ de tamaño (Sx1) outputs. Por lo tanto, la matriz X $(\mathrm{MxN})$ inputs y la matriz $\mathrm{Y}(\mathrm{SxN})$ outputs representan los datos para el total de regiones analizadas. La representación del modelo como un problema de optimización lineal, asumiendo rendimientos constantes de es- cala, se puede expresar de la siguiente forma (modelo DEA-CCR):

$$
\begin{array}{r}
\text { Min }_{\phi, \lambda} \phi \\
\text { S.a }-\mathrm{y}_{\mathrm{i}}+\mathrm{Y} \lambda \geq 0 \\
\phi \mathrm{x}_{\mathrm{i}}-\mathrm{X} \lambda \geq 0 \\
\lambda \geq 0
\end{array}
$$

donde el término $\phi$ es un escalar que multiplica al vector de inputs y representa el factor que pondera los inputs de la región evaluada, $\mathrm{x}_{\mathrm{i}}$ refiere los inputs de la i-ésima región a ser evaluada, $\mathrm{y}_{\mathrm{i}}$ representa los outputs de la i-ésima región a ser evaluada, $X$ es la matriz de inputs $\mathrm{MxN}, \mathrm{Y}$ es una matriz de outputs $\mathrm{SxN}, \lambda$ es un vector de constantes $\mathrm{Nx} 1$ que multiplica a la matriz $\mathrm{X}$ e $\mathrm{Y}$-que describe la importancia de las regiones tomadas en consideración para determinar el producto virtual o unidad de referencia, y que sirve de comparación para evaluar la i-ésima región; por último, $\mathrm{N}$ es el número de regiones. Debemos decir que el problema dual permite ilustrar acerca de la naturaleza de la eficiencia relativa, dado que se obtiene, en el caso de que existan, las holguras (slacks) o reducciones no radiales de inputs ${ }^{(14)}$. Para que una unidad sea eficiente, $\phi$ será igual a 1 y las holguras serán igual a 0 , esto es, la unidad observada se encontrará produciendo en la frontera óptima de producción ${ }^{(15)}$.

Con el fin de calcular la eficiencia técnica pura, modificamos el planteamiento anterior para incluir la posibilidad de rendimientos variables de escala:

$\begin{aligned} & \text { Min }_{\phi, \lambda} \phi \\ & \text { S.a }-\mathrm{y}_{\mathrm{i}}+\mathrm{Y} \lambda \geq 0 \\ & \phi \mathrm{x}_{\mathrm{i}}-\mathrm{X} \lambda \geq 0 \\ & \mathrm{~N} 1^{\prime} \lambda=1 \\ & \lambda \geq 0\end{aligned}$

En este modelo se incluye una restricción de convexidad ( $\mathrm{N} 1$ ' $\lambda=1$ ), donde $\mathrm{N} 1$ es vector unitario Nx1. Esta modificación permite descomponer la eficiencia en dos: por un lado, la eficiencia técnica pura (ETP), calculada 
Tabla 1. Rendimiento a escala constante [AED-CCR]. Orientación inputs.

\begin{tabular}{|c|c|c|c|c|c|c|c|c|c|c|}
\hline Año & 1997 & 1998 & 1999 & 2000 & 2001 & 2002 & 2003 & 2004 & 2005 & 2006 \\
\hline Regiones & Puntuación & Puntuación & Puntuación & Puntuación & Puntuación & Puntuación & Puntuación & Puntuación & Puntuación & Puntuación \\
\hline Región II & $97,1 \%$ & $92,8 \%$ & $92,9 \%$ & $88,3 \%$ & $86,1 \%$ & $87,8 \%$ & $100,00 \%$ & $87,3 \%$ & $91 \%$ & $90,8 \%$ \\
\hline Región III & $99,0 \%$ & $100,00 \%$ & $98,4 \%$ & $92,1 \%$ & $85,0 \%$ & $86,5 \%$ & $84,3 \%$ & $82,5 \%$ & $89,5 \%$ & $89,9 \%$ \\
\hline Región IV & $100,00 \%$ & $97,2 \%$ & $97,1 \%$ & $88,9 \%$ & $91,9 \%$ & $91,5 \%$ & $96,0 \%$ & $95,4 \%$ & $96,1 \%$ & $100,00 \%$ \\
\hline Región V & $77,5 \%$ & $74,8 \%$ & $90,5 \%$ & $76,9 \%$ & $91,3 \%$ & $74,7 \%$ & $78,3 \%$ & $78,3 \%$ & $78,2 \%$ & $80,7 \%$ \\
\hline $\mathrm{RM}$ & $68,9 \%$ & $73,8 \%$ & $83,5 \%$ & $80,8 \%$ & $84,8 \%$ & $84,9 \%$ & $86,6 \%$ & $88,1 \%$ & $89,8 \%$ & $91,1 \%$ \\
\hline Región VI & $95,7 \%$ & $91,9 \%$ & $90,5 \%$ & $90,1 \%$ & $90,3 \%$ & $79,7 \%$ & $82,9 \%$ & $82,2 \%$ & $84,1 \%$ & $83,3 \%$ \\
\hline Región VII & $100,00 \%$ & $99,2 \%$ & $97,2 \%$ & $87,0 \%$ & $88,6 \%$ & $78,1 \%$ & $79,4 \%$ & $85,5 \%$ & $83,7 \%$ & $83,3 \%$ \\
\hline Región VIII & $100,00 \%$ & $99,6 \%$ & $100,00 \%$ & $93,1 \%$ & $100,00 \%$ & $99,8 \%$ & $89,1 \%$ & $91,1 \%$ & $90,5 \%$ & $89,8 \%$ \\
\hline Región IX & $100,00 \%$ & $97,7 \%$ & $100,00 \%$ & $92,8 \%$ & $95,7 \%$ & $83,6 \%$ & $84,0 \%$ & $85,5 \%$ & $87,2 \%$ & $90,2 \%$ \\
\hline Regios X & $95,4 \%$ & $97,6 \%$ & $97,1 \%$ & $87,8 \%$ & $91,2 \%$ & $82,1 \%$ & $82,6 \%$ & $85,5 \%$ & $86,8 \%$ & $86,0 \%$ \\
\hline Región XI & $80,3 \%$ & $94,7 \%$ & $86,6 \%$ & $88,3 \%$ & $87,7 \%$ & $79,3 \%$ & $78,8 \%$ & $76,7 \%$ & $72,6 \%$ & $77,7 \%$ \\
\hline Región XII & $74,5 \%$ & $71,1 \%$ & $71,1 \%$ & $66,4 \%$ & $57,4 \%$ & $61,1 \%$ & $66,2 \%$ & $70,1 \%$ & $69,3 \%$ & $68,1 \%$ \\
\hline Total & $1088,6 \%$ & $1090,6 \%$ & $1105,2 \%$ & $1032,8 \%$ & $1050,1 \%$ & $990,2 \%$ & $1008,5 \%$ & $1008,3 \%$ & $1019,0 \%$ & $1031,3 \%$ \\
\hline Media & 0,9 & 0,91 & 0,9 & 0,8 & 0,9 & 0,8 & 0,8 & 0,8 & 0,8 & 0,9 \\
\hline MTI & 0.86081 & 0.9006182 & 0.8228818 & NUE & 0.863764 & NUE & 0.825909 & NUE & NUE & 0.846645 \\
\hline $\begin{array}{l}\text { Reducción* } \\
\text { Inputs }\end{array}$ & 0.1 & 0.01 & 0.2 & & 0.1 & & 0.2 & & & 0.1 \\
\hline
\end{tabular}

ETG: Eficiencia técnica global. MTI: Media técnicamente eficiente. NUE: No hay unidades eficientes.

Fuente: Elaboración propia a partir de datos proporcionados por el programa EMS (efficiency measurement system).

bajo el método BCC, y por otro lado la eficiencia de escala, que es el resultado del cociente de los coeficientes de eficiencia calculados con el modelo CCR y el modelo BCC. Cabe señalar que, si existen diferencias entre las dos mediciones de eficiencia para una unidad (en nuestro caso regiones), entonces significa que dicha región posee ineficiencia de escala. Formalmente, podemos determinar residualmente la eficiencia a escala como:

$$
E\left(x_{i}, y_{i}\right)=\frac{\operatorname{ETG}\left(x_{i}, y_{i}\right)}{\operatorname{ETP}\left(x_{i}, y_{i}\right)}
$$

donde ETG es la eficiencia técnica global calculada mediante el método DEA-CCR, ETP calculada mediante el método DEA-BCC; $x_{i}$ e $y_{i}$ son los inputs de la unidad $i$ y los outputs de la unidad $i$, respectivamente.

\section{RESULTADOS}

Se aplicó los modelos AED descritos en la sección anterior a los hospitales de las once regiones del país más la región metropolitana, a lo largo del período comprendido entre 1997 y 2006, con el fin de calcular la eficiencia técnica global (AED-CCR, tabla 1) y la eficiencia a escala (tabla 2). De acuerdo a la tabla 1, en el año 1997, solamente las regiones IV, VII, VIII y IX son calificadas de eficientes.

El resto de las regiones son calificadas como ineficientes técnicamente, al obtener una puntuación de eficiencia inferior a la unidad (100\%), esto es: la región II $(97,1 \%)$, región III (99\%), región $\mathrm{V}(77,5 \%)$, RM $(68,9 \%)$, región $\mathrm{X}$ $(95,4 \%)$, región XI $(80,3 \%)$, región XII $(74,6 \%)$. Un análisis individual de este año vendría a significar que, por ejemplo, los hospitales de la región $\mathrm{V}$ podrían haber reducido inputs (médicos, enfermeras, matronas, camas disponibles) en un 22,5 por 100 , para situarse sobre la frontera eficiente. Los comentarios se extienden a las demás regiones del país de ese mismo año. Una lectura más global nos indica que, en ese mismo año, el valor medio del índice de eficiencia técnica para el conjunto de la muestra alcanzó un valor de 0,9. Si consideramos las regiones técnicamen- te ineficientes, es decir, los que no están sobre la frontera, el valor medio del indicador se reduce a 0,86 , lo que implica que como promedio estos necesitarían reducir el uso de sus inputs (médicos, enfermeras, matronas, camas disponibles) en un 14 por 100, para situarse en la frontera eficiente. El mismo análisis es extensible para los otros años. La figura 1 nos muestra la tendencia de la eficiencia de las regiones a lo largo de toda la serie temporal. La duodécima región es la que presentó peores índices de eficiencia a lo largo de toda la serie temporal, en contraposición a la primera región, con mayores índices de eficiencia. Gráficamente, también se observa que el mejor año a nivel nacional fue en 1999, con un valor medio (valor unidades eficientes más ineficientes) de 0,97 por 100 .

La principal fuente de ineficiencia es la relacionada con la de escala (tabla 2 ); esta se origina cuando la entidad produce por debajo o por encima de su capacidad productiva y tiene lugar cuando el valor de la ETG (eficiencia técnica global) es menor que el valor de la ETP (eficiencia técnica pura). 
Tabla 2. Eficiencia a escala.

\begin{tabular}{|c|c|c|c|c|c|c|c|c|c|c|c|}
\hline Año & \multicolumn{1}{|c}{1997} & \multicolumn{2}{c}{1998} & \multicolumn{2}{c}{2000} & 2001 & 2003 & 2004 \\
\hline Regiones & Puntuación & Puntuación & Puntuación & Puntuación & Puntuación & Puntuación & Puntuación & Puntuación & Puntuación & Puntuación \\
\hline Región II & $99,9 \%$ & $98,8 \%$ & $98,7 \%$ & $98,5 \%$ & $97,5 \%$ & $97,3 \%$ & $100 \%$ & $97,2 \%$ & $97,3 \%$ & $97,3 \%$ \\
\hline Región III & $99,0 \%$ & $100 \%$ & $98,4 \%$ & $97,1 \%$ & $94,3 \%$ & $94,2 \%$ & $94,4 \%$ & $94,4 \%$ & $94,7 \%$ & $95,2 \%$ \\
\hline Región IV & $100 \%$ & $98,5 \%$ & $97,1 \%$ & $96,1 \%$ & $97,8 \%$ & $99,9 \%$ & $99,4 \%$ & $99,2 \%$ & $99,2 \%$ & $100 \%$ \\
\hline Región V & $99,6 \%$ & $99,8 \%$ & $99,2 \%$ & $99,7 \%$ & $99,3 \%$ & $99,3 \%$ & $99,1 \%$ & $99,2 \%$ & $99,2 \%$ & $98,6 \%$ \\
\hline RM & $77,0 \%$ & $80,9 \%$ & $83,5 \%$ & $80,8 \%$ & $84,8 \%$ & $84,9 \%$ & $87,3 \%$ & $88,1 \%$ & $89,8 \%$ & $91,1 \%$ \\
\hline Región VI & $99,1 \%$ & $99,1 \%$ & $98,2 \%$ & $98,3 \%$ & $99,1 \%$ & $98,3 \%$ & $98,8 \%$ & $98,5 \%$ & $98,6 \%$ & $98,7 \%$ \\
\hline Región VII & $100 \%$ & $99,8 \%$ & $99,1 \%$ & $99,1 \%$ & $98,7 \%$ & $99,4 \%$ & $99,8 \%$ & $99,7 \%$ & $99,5 \%$ & $99,7 \%$ \\
\hline Región VIII & $100 \%$ & $99,9 \%$ & $100 \%$ & $99,9 \%$ & $100 \%$ & $99,8 \%$ & $98,6 \%$ & $98,3 \%$ & $98,2 \%$ & $98,3 \%$ \\
\hline Región IX & $100 \%$ & $99,2 \%$ & $100 \%$ & $99,5 \%$ & $95,7 \%$ & $99,3 \%$ & $99,8 \%$ & $99,8 \%$ & $99,9 \%$ & $99,8 \%$ \\
\hline Región X & $99,4 \%$ & $99,9 \%$ & $99,7 \%$ & $99,9 \%$ & $99,5 \%$ & $98,5 \%$ & $99,2 \%$ & $99,6 \%$ & $99,4 \%$ & $99,4 \%$ \\
\hline Región XI & $80,3 \%$ & $94,7 \%$ & $86,5 \%$ & $88,3 \%$ & $87,7 \%$ & $86,1 \%$ & $86,7 \%$ & $88 \%$ & $85,7 \%$ & $87,6 \%$ \\
\hline Región XII & $74,5 \%$ & $71,0 \%$ & $71,1 \%$ & $66,4 \%$ & $87,6 \%$ & $74,6 \%$ & $83,8 \%$ & $90,6 \%$ & $90,5 \%$ & $90,7 \%$ \\
\hline Total & $1129,1 \%$ & $1142,2 \%$ & $1131,9 \%$ & $1123,9 \%$ & $1142,2 \%$ & $1131,9 \%$ & $1147,2 \%$ & $1152,9 \%$ & $1152,3 \%$ & $1156,65 \%$ \\
\hline Media & 0,9 & 0,9 & 0,9 & 0,9 & 0,9 & 0,9 & 0,9 & 0,9 & 0,9 & 0,9 \\
\hline
\end{tabular}

Fuente: Elaboración propia, a partir de datos proporcionados por el programa EMS (efficiency measurement system).

Observemos que hay regiones que presentan eficiencia a escala (valor igual a 1 o 100 por ciento), mostrando de esta forma que el resto de las regiones no estuvieron operando a escala óptima. La importancia de llevar a cabo un análisis de eficiencia de las unidades radica en la necesidad de determinar cuáles de las regiones presentan un mejor desempeño y cuáles pueden mejorar el uso de sus recursos.

\section{DISCUSIÓN}

El análisis AED es una buena herramienta para llevar a cabo mediciones de eficiencia y evaluar resultados de gestión, pues permite conocer aquellas unidades que realizan la mejor asignación de sus recursos en comparación al resto de la muestra. Para nuestro análisis, hemos creído conveniente utilizar la metodología no-paramétrica análisis envolvente de datos (AED), frente a otras alternativas del análisis paramétrico. La técnica no paramétrica nos da la ventaja de ser más flexible, ya que no se requiere especificar alguna forma funcional entre las variables, lo cual constituye una gran ventaja en esta investigación.
En este trabajo hemos analizado la eficiencia técnica de un conjunto de hospitales regionales en un período de 10 años. Los resultados del estudio sugieren que a lo largo de la serie solamente algunas regiones han hecho un uso eficiente de los recursos, mientras otras regiones del país han quedado fuera de la frontera eficiente. En particular, el año 1997 fue el año de mejor desempeño. En dicho año se detectó cuatro regiones técnicamente eficientes, esto es, las regiones IV, VII, VIII, IX. En promedio estas mismas regiones hicieron, a lo largo de toda la serie temporal, un empleo adecuado de sus recursos. Por otro lado, aquellos hospitales regionales que no hicieron uso adecuado de los recursos, tendrían que reducir sus inputs en un $10 \%$ en promedio para situarse en la frontera eficiente. Esta ineficiencia podría estar explicada, por ejemplo, por el sobredimensionamiento del recurso humano y el sobrecosto de los mismos.

Cabe señalar que los resultados del análisis no son muy robustos, por cuanto nos hemos encontrado con una serie de limitaciones que tienen que ver con el acceso a una base de datos más desagregada, que nos permitiera analizar no solo la eficiencia técnica a nivel hospitalario sino también los factores asociados a dicha ineficiencia. Por otro lado, es necesario insistir que en el análisis no hemos podido cuantificar los recursos desperdiciados de las unidades ineficientes detectadas. Creemos que este trabajo es una primera aproximación del análisis de eficiencia hospitalaria en Chile.

\section{AGRADECIMIENTOS}

En la elaboración de este trabajo agradezco al Ministerio de Salud de Chile (MINSAL) el acceso a la base datos en papel y versión electrónica.

\section{REFERENCIAS BIBLIOGRÁFICAS}

1. Garcia B. Análisis de eficiencia del sector hospitalario: una revisión de métodos. Cuadernos de estudios empresariales. 1997;7:151-76.

2. Salas V. Prólogo. En: Prior D, Solá M. La eficiencia de los hospitales de Cataluña. Comparación entre los hospitales públicos y privados. Barcelona, Fulls Economics, Generalitat de Catalunya, Departament de Sanitat y Seguretat Social. 1993.

3. Nyhan R, Cruise P. Comparative performance assessment in managed care: data envelopment analysis for health care managers. Manag Care Q. 2000;8(1):18-27.

4. Reyes M, Figueroa E, Córdoba JA, López FJ. Análisis envolvente de datos: aplicación en la medida de la eficiencia en los servicios de medicina 
preventiva hospitalaria de Andalucia. Rev San Hig Púb. 1993;67:455-64.

5. Ligarda J, Naccha M. La eficiencia de las organ izaciones de salud a través del análisis envolvente de datos. Microrredes de la dirección de salud IV Lima Este. An Fac med. 2006;67(2):142-51.

6. Nayar P, Ozcan YA. Data envelopment analysis comparison of hospital efficiency and quality. J Med Syst. 2008;32(3):193-9.

7. Andes S, Metzger LM, Kralewski J, Gans D. Measuring efficiency of physician practices using data envelopment analysis. Manag Care. 2002;11(11):4854.

8. Puig-Junoy J. ¿Qué sabemos acerca de la eficiencia de las organizaciones sanitarias en España? Avances en la gestión sanitaria: implicaciones para la política, las organizaciones sanitarias y la práctica clínica. Barcelona (España): Ed. Asociación de Economía de la Salud. 2000.

9. Chilingerian JA, Sherman HD. DEA and primary care physician report cards: Deriving preferred practice cones from managed care service concepts and operating strategies. Annals of Operations Research. 1997;73:35-66

10. Gorgemans S. Eficiencia y dependencia funcional evidencia en el sector hospitalario publico. XIV encuentro de economía pública. 2002

11. Farrell MJ. The measurement of productive efficiency. Journal of the Royal Statistical Society, Series A. 1957;120(A):253-90.

12. Banker RD, Charnes A, Cooper WW. Some models for estimating technical and scale efficiencies in data envelopment analysis. Manag Sci. 1984;30(9):1078-92.
13. Banker R. Maximum likelihood, consistency and data envelopment analysis: statistical foundation. Manag Sci. 1993;39:1265-73.

14. Charnes A, Cooper W, Rodhes E. Measuring the efficiency of decision-making units. Eur J Operat Res. 1978;2:429-44.

Trabajo recibido el 30 de noviembre de 2010 y aceptado para publicación el 27 de diciembre de 2010

Correspondencia:

Correo electrónico: planck.barahona@uda.cl. 